\title{
Research on Unwrapping Algorithm of Concentric Annular Image
}

\author{
Zhang Sheng ${ }^{1, \mathrm{a}}$, Lin Ming-xing ${ }^{1, \mathrm{~b} *}$ and Wang Qing-dong ${ }^{1, \mathrm{c}}$ \\ ${ }^{1}$ Key Laboratory of Ministry of Education, School of Mechanical Engineering, \\ Shandong University, 17923 Jingshi Road, Jinan, 250061, P.R. China

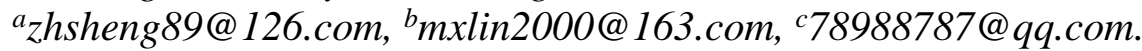

\begin{abstract}
To meet the demand of rapid, accurate and online inspection of precision parts' defects, the CCD camera is used to collect information of the inner hole. Since the obtained source image is a concentric annular image of 360 degrees with large image distortion, meanwhile the collected source images are not suitable to be processed directly with the image processing technology. So further processing is needed to be done for the rectangle unwrapping images. The method of Hough transform is adopted in this paper to find the center of a circle and the corresponding position used the center as a reference. On the basis of positioning the circle center, a kind of circular image unwrapping algorithm is designed by the transformational relation between Cartesian and polar coordinate. During unwrapping of the image, the bilinear interpolation algorithm is adopted to improve the image quality when the image unwrapping algorithm is used.

The algorithm is verified by program and the result indicates that the algorithm to unwrap the image is efficient in locating the center by Hough transform combined with the coordinate's transformation and the bilinear interpolation. Furthermore, the algorithm can unfold the image accurately and quickly and ensure the quality of the image, which prepares the foundation for subsequent processing.
\end{abstract}

Keywords: Image distortion; Unwrapping algorithm; Hough transform; Bilinear interpolation

\section{Introduction}

To ensure machining quality, the defect on inner surface of parts needs to be detected in many industry and engineering production areas [1-4]. The ways to detect the inner surface of parts has been widely used at home and abroad, which is mainly divided into two main types: sub-contact detection and non-contact detection [5]. In recent years, with the development of computer and image processing techniques, more and more defect detections of the inner surface of parts have been made by using the image processing techniques. The image processing technology belongs to non-contact detection, which has no effect on inner surface of the parts, ensures the integrity of precision parts and avoids the unnecessary damage caused by the contact of the detection process.

The valve sleeve of the car steering is a core component of the automotive steering systems. The fitting clearances of the valve sleeve and plug is designed between 0.010 and $0.014 \mathrm{~mm}$, which requires the valve sleeve inner hole should have a high dimensional accuracy, geometric accuracy and minimal roughness. Therefore, it is necessary to detect the defect on the inner hole of the valve sleeve. The defect detection is done manually now, thus wastes a lot of time and energy, furthermore the detection accuracy cannot be

${ }^{*}$ Corresponding Author 
guaranteed. So the machine vision is used in this paper to detect the defect on the inner hole surface of the valve sleeve.

The CCD camera can acquire all the inner surface information of the parts one time for small structures. And its advantages such as good flexible design, no scanning, and no stitching can lessen the workload caused by multiple extractions and save the extraction time [6]. However, the obtained image is a 360 degree panorama circular one due to the structural features of the CCD camera, which is not comfortable to be observed by eyes. The rectangle unwrapping is needed to make the defect easy to be inspected and further processed [7].

The following two unwrapping algorithms are generally used: First, the image is unwrapped with coordinate transformation relations directly, and the pixels compensated with neighbor interpolation algorithm in the process of unwrapping [8]. Because it has fewer calculation and simple mutual relation, the neighbor interpolation algorithm is widely used in practice. However, it leads to serious pixels loss and ineffective unwrapping due to the simple roundness. Second, the neighbor interpolation algorithm is improved because the pixels loss problem caused by the simple round numbers in the neighbor interpolation algorithm is solved by compensating the pixels with bilinear interpolation algorithm in the process of unwrapping [9]. Because there isn't a unified center positioning reference, unwrapping from the eccentric dot in the process of unwrapping leads to the irregular head face and brings problems to the subsequent processing.

This paper comes up with a method using the bilinear interpolation algorithm to unwrapping the image to avoid the mentioned shortcomings. The Hough transform searches the center of the source image before unwrapping, upon which a standard positioning is set for the source image and a polar coordinate system of the source images at the center point is established. In the process of unwrapping, the corresponding location is alternated with the transformational relation between Cartesian and polar coordinates [10].

The pixel value of the target image is obtained from the bilinear interpolation of the four pixels of the source image, which will solve the problem of pixels loss caused by the simple roundness [11]. Meanwhile, the end surface of the unwrapping image is regular for the subsequent processing when the Hough transform is applied to achieve the center position. It is verified by the experiment that the method can expand panoramic circular image of the 360 degrees into the rectangle image rapidly and accurately and has a wide application prospect.

\section{Extract Origin of the Panoramic Circular Image}

The panoramic annular image collected by the CCD camera is showed in Figure 1. The extract of the central point of the panoramic circular image is the most fundamental step in the entire image unwrapping algorithm: all the following algorithms are based on the selected central point. Therefore, it becomes more important to seek the center accurately. 


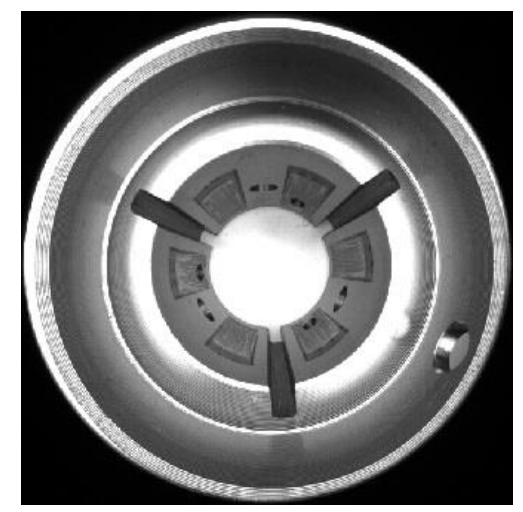

Figurer 1. The Panoramic Circular Image

It is a very mature algorithm by the Hough transform for circle extraction. Meanwhile, it has prominent advantages of robustness for the segmentation result and insensitive to noise pollution, so the Hough transform is appropriate for extracting center coordinates [12]. The radius of a circle $r$ can also be achieved after finding out the center of the panoramic circular image using Hough transform. Supposing some point in the circle is the circle pixel, and a circle is drawn from this pixel point as circle center, $\mathrm{r}$ as radius, it will gain a track probably passing through the circle center. After the operation mentioned above is repeated for many times, the pixel with the highest occurrence frequency will be the circle center. The center coordinates can be got accurately, even through the circles are lost or overlap in the process of the pretreatment.

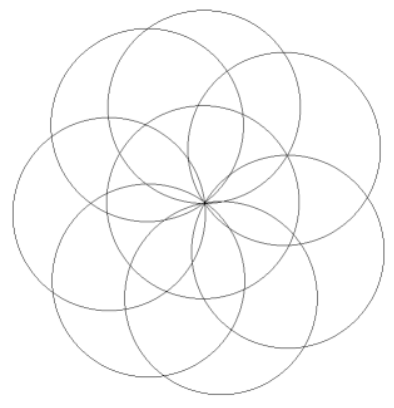

Figure 2. The Theory of Hough Transforms

\section{Application of the Bilinear Interpolation Algorithm}

The most common interpolation algorithms for unwrapping image are the nearest neighbor interpolation algorithm and the bilinear interpolation algorithm. Owing to the simpler the interpolation, the larger the loss will be in terms of geometry and the computational accuracy of the gray value [13]. Bilinear interpolation algorithm is used to ensure the accuracy of the image unwrapping. The bilinear interpolation makes the twodimensional linear interpolate calculation based on the gray value of the most proximal 4 points around the pixels. Therefore, the bilinear interpolation algorithm has no boundary gradient problem, can also retain the details of the image well [14].

Bilinear interpolation uses the bicubic polynomial:

$$
\begin{aligned}
& a_{00}+a_{10} x+a_{01} y+a_{20} x^{2}+a_{11} x y+a_{02} y^{2}+a_{21} x^{2} y+a_{12} x y^{2}+a_{30} x^{3}+a_{03} y^{3} \\
& +a_{22} x^{2} y^{2}+a_{13} x y^{3}+a_{31} x^{3} y+a_{32} x^{3} y^{2}+a_{23} x^{2} y^{3}+a_{33} x^{3} y^{3}
\end{aligned}
$$

Another manifestation of the cubic polynomial: 


$$
\sum_{i=0}^{3} \sum_{j=0}^{3} a_{i j} x^{i} y^{j}
$$

The parameter value $a_{\mathrm{ij}}$ depends on the characteristic of the difference data, if the derivatives of interpolating function have already been known, the coordinates with four endpoints and from the first to the third derivatives of each endpoint will be used usually.

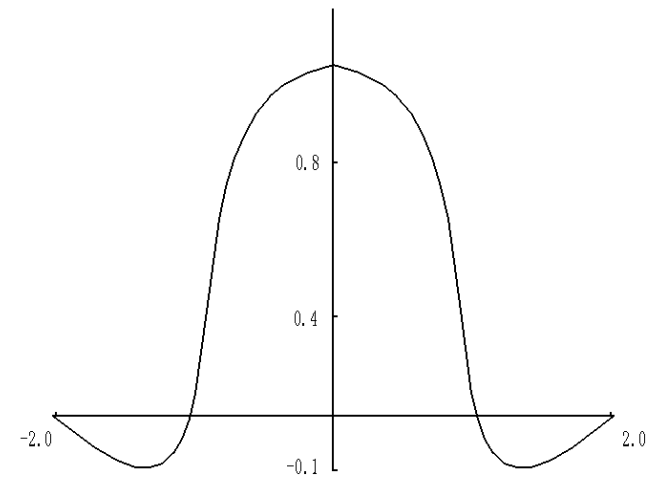

Figure 3. Kernel of One-dimensional Interpolation

One-dimensional interpolation kernel shown in the Figure 3, the formula is:

$$
\mathrm{S}(x)=\left\{\begin{array}{cc}
1-2|x|^{2}+|x|^{3} & 0 \leq|x| \leq 1 \\
4-8|x|+5|x|^{2}-|x|^{3} & 1 \leq|x| \leq 2 \\
0 & \text { else }
\end{array}\right.
$$

In this formula $|x|$ is the distance between the pixels and the center point is along the horizontal ordinate. The pixel gray value in the interpolation $(x, y)$ can be worked out by weighted interpolation of gray values of the around 16 pixels.

Formula as follows:

$$
f(x, y)=f(i+u, j+v)=A B C
$$

Among them:

$$
\begin{aligned}
\mathrm{A} & =\left[\begin{array}{l}
S(1+v) \\
S(v) \\
S(1-v) \\
S(2-v)
\end{array}\right]^{T} \quad C=\left[\begin{array}{l}
S(1+u) \\
S(u) \\
S(1-u) \\
S(2-u)
\end{array}\right]^{T} \\
B & =\left[\begin{array}{llll}
f(i-1, j-1) & f(i-1, j) & f(i-1, j+1) & f(i-1, j+2) \\
f(i, j-1) & f(i, j) & f(i, j+1) & f(i, j+2) \\
f(i+1, j-1) & f(i+1, j) & f(i+1, j+1) & f(i+1, j+2) \\
f(i+2, j-1) & f(i+2, j) & f(i+2, j+1) & f(i+2, j+2)
\end{array}\right]
\end{aligned}
$$




\section{Unwrapping Image of the Inner Hole}

The extraction of the exact center point before the inner hole unwrapping and the bilinear interpolation are the preparations for the image unwrapping algorithm of this paper. The inner hole image of the parts collected by the CCD camera is as shown in the Figure 1. The polar coordinate system is established at the center of a circle found in the Hough transform through the parts inner hole collected by the CCD camera. The coordinate transformation relation between Cartesian and polar coordinates is used and supposing expanding from $o b$ axis in the source image, firstly, set the coordinates of the pixel to be displayed, then, alternate the relation according to the coordinate, find the corresponding pixels coordinates in the source image.

The panoramic circular image is transformed in proper order from left to right and bottom to top. The corresponding transformational coordinate value can be got pixels on the concentric circles in the image. The corresponding transformational coordinate value can be got through pixels on the concentric circles in the image by increasing radius ceaselessly and setting some angle or radian as a step [14]. As shown in the Figure 4: Establish the panoramic circular image coordinate system $a-b$ in which $O$ as center, $r$ as radius, $\mathrm{R}$ as external diameter, the annulus part in the middle is the one need to be expanded. Set the expanding standard radius as $R^{\prime}$.

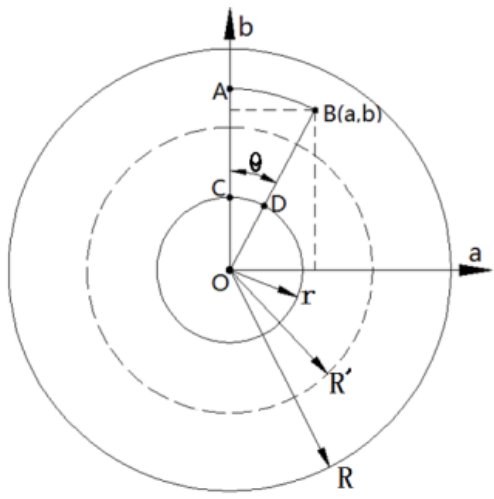

Figure 4. Polar Coordinates of the Panorama Image

According to the transform relation between Cartesian and polar coordinates, the expanded target image is shown in the Figure 5. The point $O$ in the coordinate corresponds the point $\mathrm{C}$ in the source image. Supposing the selected point in the source image is $B(a, b)$, corresponding point is $P(x, y)$ in the target image, the correspondence need to be found between the two functions: $x=F_{u n}(a, b)$ and $y=F_{u n}(a, b)$.

The following is the inference: according to the source image coordinate system:

$a=\overline{B O} \cdot \sin \theta$

$b=\overline{B O} \cdot \cos \theta$

$\overline{B O}=r+\overline{B D}$

The unwrapping image shows:

$\overline{B D}=y$ 


$$
\theta=x / R
$$

Summarize the above formula can obtain:

$$
\begin{aligned}
& \theta=\arctan (a / b) \\
& x=R^{\prime} \cdot \arcsin (a / b) \\
& y=a / \sin \theta-r
\end{aligned}
$$

Finally the corresponding relationship between the target coordinate system and the source coordinate system is obtained, and then the expansion operation will be done according to it.

In the process of the expansion, the length of a circle remains unchanged without compressing or stretching, namely expansion basic circle with the radius is $R^{\prime}$. The phenomenon of recurrence and the pixel loss may appear during expansion: When expanding the image, the image information shorter than radius $R^{\prime}$ of the basic circle is stretched horizontally, the one longer than radius $R^{\prime}$ of the basic circle is compressed horizontally [15]. Therefore, the bilinear interpolation algorithm is adopted in the area where need to be stretched or compressed to make up the reiteration of the pixel and the loss problem.

Finally, the expansion length expanded by the bilinear interpolation in the target image is: $L=\overline{O M}=2 \pi R^{\prime}$, the width is that the big circle radius minus small round radius, namely: $W=\overline{O N}=R-r$. The choice of the basic radius $R^{\prime}$ is based on the aspect ratio and practical effect, here $R^{\prime}=(R+r) / 2$.

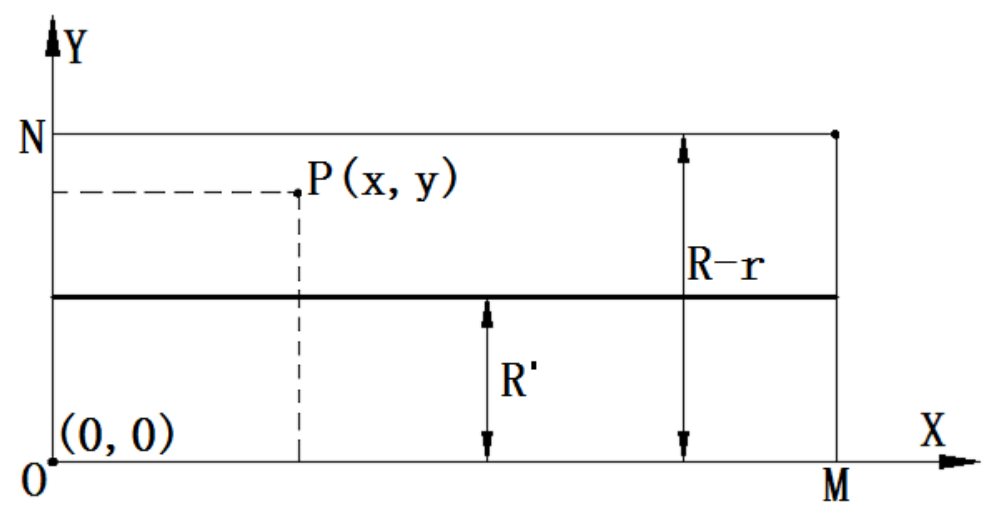

Figure 5. The Expanded Target Image

\section{Verify the Algorithm}

The Visual Studio $\mathrm{C}++$ programming is used to validate the unwrapping algorithm in order to verify its feasibility. The input image is the source image in Figure 2. The expansion histogram is shown in the Figure 6, to increase the comparability it is supposed that the center location hasn't been changed by the Hough transform. The upper surface expanded is non-linear and has some distortions due to the eccentricity in the process of the expansion, which has an impact on the subsequent processing work.

The unwrapping rectangle image based on the center location of the Hough transform is shown in the Figure 7, it can be seen from the image that the method can make up perfectly the shortcoming of irregularity of the end face brought by the eccentricity 
expansion. Also, the distortion is very small after the image expansion, which paves the way for further processing.

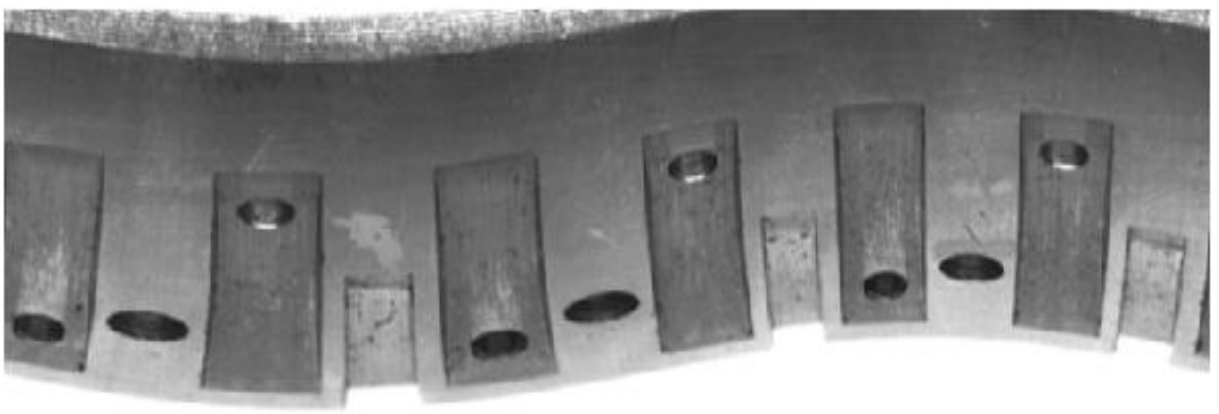

Figure 6. Unwrapping Image without Hough Transform Positioning

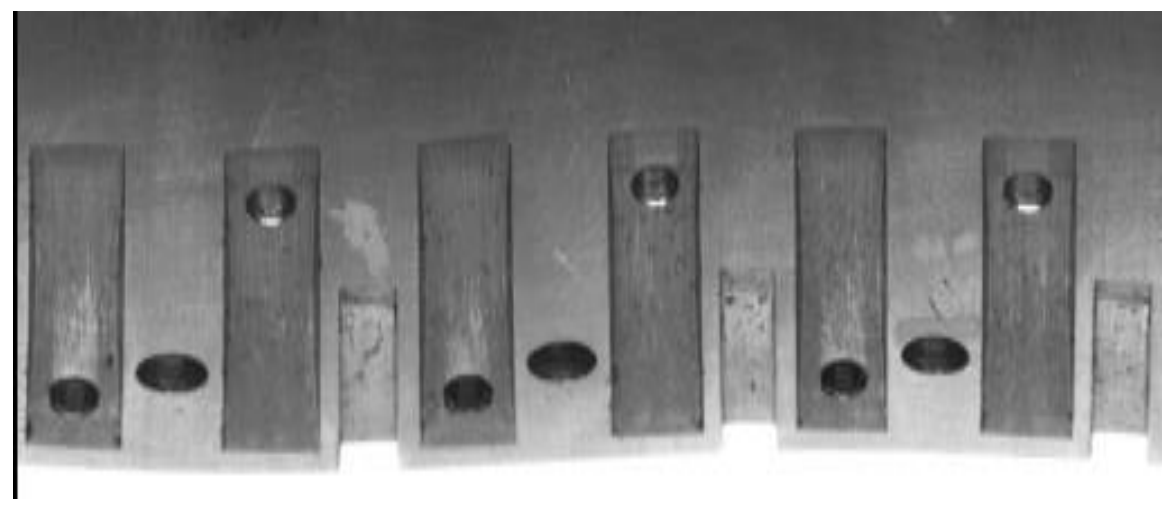

Figure 7. Unwrapping Image with Hough Transform Positioning

\section{Conclusions}

It is verified that the algorithm can make the original image unwrapping quick and accurate, and it provides a good foundation for further processing. The unwrapping algorithm put forward in this paper has a good practicability. However, large amount of calculations need to be done in the process. In addition, the unwrapping effect of the image should be further improved, and amount of calculation should be further optimized in subsequent research.

\section{Acknowledgment}

This work is supported by Shandong Province Science and Technology Development Project (NO.2014GGX103025).

\section{References}

[1] Miyazawa and Takashi, "Defect Detecting Method and Apparatus", Google Patents, (1984) July 16.

[2] M. Miyashita, M. Kageyama and H. Hiratsuka, "Method and Apparatus for Detecting Defect on Semiconductor Substrate surface", Google Patents, (1993) April 16.

[3] S. Yan, P. C. Wu and T. J. Lu, "Nondestructive Testing Technique for Strip Surface Defects and Its Application", Nondestructive Testing, (2000), pp. 78-81.

[4] C. Gao, K. Ren and Y. C. Guo, "Surface Crack Examination Technology Based on Machine Vision", Aviation Precision Manufacturing Technology, vol. 23, no. 5, (2007), pp. 014-016.

[5] T. Ling, X. Zhao and Z. Hou, "Fast Panoramic Annular Image Stretching Based on CUDA", Computer Technology and Development, vol. 27, no. 4, (2011), pp. 008-012.

[6] W. Fischer, G. Caprari and R. Siegwart, "Foldable Magnetic Wheeled Climbing Robot for the Inspection of Gas Turbines and Similar Environments with Very Narrow Access Holes", Industrial Robot: An International Journal, vol. 24, no. 2, (2010), pp. 244-249. 
[7] Q. D. Zhu, G. L. Cheng and X. Meng, "Implementation of Fast Cylindrical Unwrapping Algorithm for Omni-directional Image Based on FPGA", Applied Science and Technology, (2011), pp. 003-005.

[8] T. Chen, D. Yang and X. Shi, "Optimization of Omnidirectional Image Unwrapping Based on Multicore Processor. Computer Engineering", vol. 13, no. 8, (2011), pp. 100-103.

[9] B. Wu, W. Han and Z. Shao, "Surface Defects Inspecting Inside Small Bores Based on Machine Vision", Journal of Optoelectronics. Laser, vol. 27, no. 2, (2012), pp. 019-022.

[10] X. Z. Bu, G. Li and B. Yang, "Fast Unwrapping of Panoramic Annular Image with Center Deviation", Optics and Precision Engineering, vol. 32, no. 5, (2012), pp. 2103-2109.

[11] Y. M. Feng, D. Y. Yang and J. Lu, "Omnidirectional Image Unwrapping Based on Quadratic Interpolation Method", Journal of Computer Engineering and Applications, vol. 25, no. 2, (2008), pp. 54-55.

[12] Z. M. Zhang and L. Zhang, "Panorama Stitching and Spread Based on Central Location", Electronic Science and Technology, vol. 56, no. 1, (2012), pp. 009-012.

[13] C. Toepfer and T. Ehlgen, "A Unifying Omnidirectional Camera Model and Its Applications in Computer Vision", 2007. IEEE 11th International Conference on, (2007), pp. 54-57.

[14] G. Cheng, Z. H. Xiong and L. D. Chen, "Real-time Unrolling Catadioptric Omni-directional Images on DSP", Journal of Computer Applications, vol. 14, no. 4, (2008), pp. 29-32.

[15] Z. Xiong, W. Xu and W. Wang, "Reduce Look-up Table Space in Panorama Unrolling of Catadioptric Omni-directional Images by Eight Direction Symmetry Reuse Strategy", Minimicro Systems-Shenyang, (2007), pp. 1832-1836.

\section{Authors}

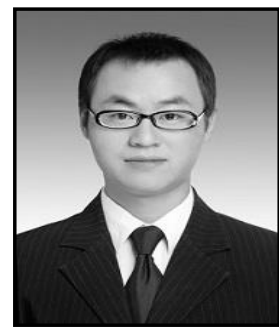

Zhang Sheng, is currently postgraduate candidate in Shandong University. His interests includes machine vision, image processing and structure design. With the guidance of my tutor, I have been the lead researcher on several studies, completed several actual production projects successfully. This paper gets financial support from the science and technology develop project in Shandong Province.

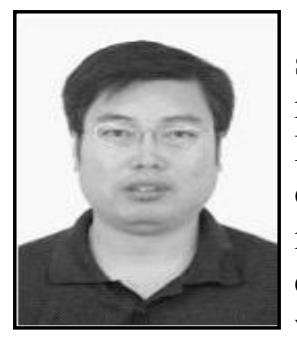

Lin Ming-xing, is currently the professor and the doctoral supervisor of mechanics engineering at Shandong University, senior member of Chinese Mechanical Engineering Society and China Instrument and Control Society, Youth Working Committee members of China Instrument and Control Society. His major research covers machine vision, image processing, intelligent measurement and control. He has published a book and some magazine articles, of which there are scores of papers indexed by EI. Also, he has carried out national fund project and some provincial-level scientific research projects. He has more than a dozen Patents for Invention and Utility Model Patents. 